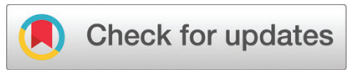

Cite this: Nanoscale, 2020, 12, 14448

Received 22nd April 2020,

Accepted 19th June 2020

DOI: 10.1039/d0nr03170d

rsc.li/nanoscale

\section{Tuning trion binding energy and oscillator strength in a laterally finite 2D system: CdSe nanoplatelets as a model system for trion properties $\uparrow$}

\author{
Sabrine Ayari, ${ }^{a}$ Michael T. Quick, ${ }^{\mathrm{b}}$ Nina Owschimikow, ${ }^{\mathrm{b}}$ Sotirios Christodoulou, (D) \\ Guillaume H. V. Bertrand, (D) ${ }^{d}$ Mikhail Artemyev, (D) ${ }^{\mathrm{e}}$ Iwan Moreels, (D) ${ }^{f}$ \\ Ulrike Woggon, ${ }^{b}$ Sihem Jaziri ${ }^{a, g}$ and Alexander W. Achtstein (iD *b
}

\begin{abstract}
We present a theoretical study combined with experimental validations demonstrating that $\mathrm{CdSe}$ nanoplatelets are a model system to investigate the tunability of trions and excitons in laterally finite 2D semiconductors. Our results show that the trion binding energy can be tuned from $36 \mathrm{meV}$ to $18 \mathrm{meV}$ with the lateral size and decreasing aspect ratio, while the oscillator strength ratio of trions to excitons decreases. In contrast to conventional quantum dots, the trion oscillator strength in a nanoplatelet at low temperature is smaller than that of the exciton. The trion and exciton Bohr radii become lateral size tunable, e.g. from $\sim 3.5$ to $4.8 \mathrm{~nm}$ for the trion. We show that dielectric screening has strong impact on these properties. By theoretical modeling of transition energies, binding energies and oscillator strength of trions and excitons and comparison with experimental findings, we demonstrate that these properties are lateral size and aspect ratio tunable and can be engineered by dielectric confinement, allowing to suppress e.g. detrimental trion emission in devices. Our results strongly impact further in-depth studies, as the demonstrated lateral size tunable trion and exciton manifold is expected to influence properties like gain mechanisms, lasing, quantum efficiency and transport even at room temperature due to the high and tunable trion binding energies.
\end{abstract}

\footnotetext{
${ }^{a}$ Laboratoire de Physique des Materiaux, Faculte des Sciences de Bizerte, Universite de Carthage, Jarzouna 7021, Tunisia

${ }^{b}$ Institute of Optics and Atomic Physics, Technische Universität Berlin, Strasse des 17. Juni 135, 10623 Berlin, Germany.E-mail: achtstein@tu-berlin.de

${ }^{c}$ ICFO-Institut de Ciencies Fotoniques, 08860 Castelldefels, Barcelona, Spain

${ }^{d}$ CEA Saclay, 91191 Gif-sur-Yvette, France

${ }^{e}$ Research Institute for Physical Chemical Problems of Belarusian State University, 220006 Minsk, Belarus

${ }^{f}$ Department of Chemistry, Ghent University, Krijgslaan 281 - S3, 9000 Gent, Belgium ${ }^{g}$ Laboratoire de Physique de la Matiere Condensee, Departement de Physique, Faculte des Sciences de Tunis, Campus Universitaire, 1060 Tunis, Tunisia

$\dagger$ Electronic supplementary information (ESI) available: Details on theoretical model, samples and experimental results. See DOI: 10.1039/D0NR03170D
}

\section{Introduction}

II-VI semiconductor nanoplatelets (NPLs) are colloidal analogues to epitaxial quantum wells, yet with a finite lateral size. $^{1-24}$ Compared to zero-dimensional (OD) quantum dots (QDs) and one-dimensional (1D) nanorods (NRs), two-dimensional (2D) zinc blende CdSe NPLs have sharper absorption and emission peaks, larger absorption cross-sections and radiative decay rates allowing high quantum yields, and they also exhibit directed and polarized emission. ${ }^{1-3,22,25-30}$ Similar to 2D transition metal dichalcogenides (TMDCs) ${ }^{31-33}$ and perovskites $^{34-36}$ showing strong confinement in $z$-direction and dielectric mismatch to the surroundings, CdSe NPL excitons exhibit high exciton binding energies making them stable even at room temperature., ${ }^{2,5,23,37-39}$ In laterally infinite TMDCs, trionic states with high binding energies have been predicted and observed. ${ }^{40-48}$ In contrast to TMDCs, there is a precise synthesis based control over the finite lateral size $e^{4,49}$ and thickness of CdSe nanoplatelets reported in ref. 50. The controlled monolayer (ML) thickness precission results in narrow PL emission. ${ }^{1,2,22}$ The excitonic optical properties and dynamics of NPLs interpolate between the Coulomb correlation- and confinement-dominated limits known from ideal quantum wells and quantum dots. As a consequence, the optical response of excitons in CdSe NPLs can be tuned strongly by the lateral size and thickness, ${ }^{2,18,51}$ as they are a system between quantum well-like (weak lateral confinement) and quantum dot-like (strong confinement) regimes. ${ }^{7,51}$ Until now, the influence of the finite lateral size on the electronic properties of trions has not been investigated comprehensively in theory and experiment.

Based on theoretical modeling of the exciton and trion energies, we investigate the properties of trions and excitons in CdSe nanoplatelets. We study the tunability of the radiative rate, oscillator strength, binding energy and Bohr radius of trions and excitons in the nanoplatelets. We show that nanoplatelets are a model system for laterally finite $2 \mathrm{D}$ semi- 


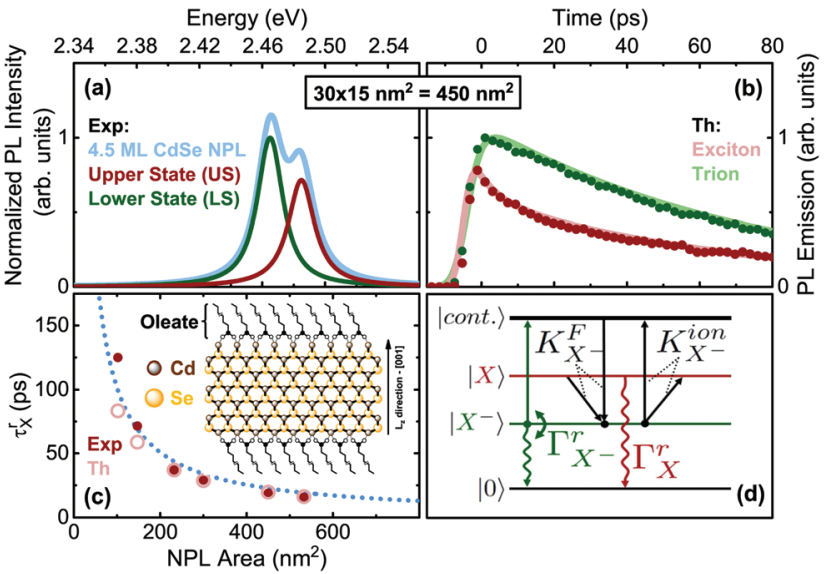

Fig. 1 (a) Time-integrated PL emission of 4.5 monolayer (ML) CdSe NPLs for exemplary lateral size at $4 \mathrm{~K}$ and $\sim 0.2 \mathrm{~W} \mathrm{~cm}^{-2}$ (CW equivalent) $420 \mathrm{~nm}$ excitation, showing a lower state (LS, trion) and upper state (US, exciton) emission. (b) Transient PL decay under the same conditions. (c) Exciton radiative rates from Table 1. Inset: Structure of CdSe NPLs with ligands. (d) Level scheme of the trion model: Crystal ground state $|0\rangle$ (no excitation), trion $\left|X^{-}\right\rangle$, exciton $|X\rangle$ and $\mathrm{e}-\mathrm{h}$ pair continuum states, as well as allowed transitions, see $\mathrm{ESI}$ section $\mathrm{S} 3 \uparrow$ for details.

conductors to study their size tunable trion and exciton physics. We show that e.g. the trion binding energy and oscillator strength can be tuned in a way that at room temperature a large fraction or no trions are present, paving the way e.g. to an effective control of the emission efficiency of devices. We substantiate our theory results by comparison with experiments.

An exemplary low temperature photoluminescence (PL) spectrum of 4.5 monolayer (ML) CdSe nanoplatelets (NPS) is shown in Fig. 1(a) along with a sketch of the chemical platelet structure (inset in c). The PL shows a double emission. ${ }^{5,14,16,17,52-55}$ (Details on the samples, preparation and time-resolved and integrated PL spectroscopy can be found in sections S2 and S3 of (ESI $\dagger$ ).) A lower state (LS) trion emission and an upper state (US) exciton emission have been identified. ${ }^{54,55}$ The energy spacing $\Delta E$ strongly depends on the lateral platelet size and varies from 38 to $18 \mathrm{meV}$, see Fig. S3 in the ESI $\dagger$ and Table 1 . We investigate in this paper the properties of trions and excitons and the tunability of their properties by the lateral size.

Table 1 Experimental fit parameters of the rate equation model for NPLs of different sizes (see section S3A of $(E S I \dagger)$ ) as well as the exciton radiative rates (theo.), as obtained from theory below

\begin{tabular}{lllllll}
\hline Size $\left(\mathrm{nm}^{2}\right)$ & $17 \times 6$ & $21 \times 7$ & $29 \times 8$ & $30 \times 10$ & $30 \times 15$ & $41 \times 13$ \\
Area $\left(\mathrm{nm}^{2}\right)$ & 102 & 147 & 232 & 300 & 450 & 533 \\
$\Delta E(\mathrm{meV})$ & 36 & 32 & 26 & 24 & 20 & 18 \\
$\Gamma_{\mathrm{X}}^{\mathrm{r}}\left(\mathrm{ns}^{-1}\right)$ & 8 & 14 & 27 & 35 & 53 & 64 \\
$\Gamma_{\mathrm{X}^{-}}{ }^{\mathrm{o}}\left(\mathrm{ns}^{-1}\right)$ & 1.6 & 1.9 & 2.5 & 3.3 & 4.1 & 4.6 \\
$\gamma_{\mathrm{X}}{ }^{-}\left(\mathrm{ns}^{-1}\right)$ & 62 & 77 & 111 & 96 & 78 & 32 \\
$\Gamma_{\mathrm{X} \text {,theo }}^{\mathrm{r}}\left(\mathrm{ns}^{-1}\right)$ & 11 & 16 & 26 & 34 & 50 & 62
\end{tabular}

\section{Results and discussion}

Charged excitons (trions) appear frequently in 2D transition metal dichalcogenides (TMDCs) and are demonstrated in CdSe platelets. $^{15,17,55}$ For a negative trion, the two opposite-spin electrons of an $X^{-}$trion can be in a triplet state $S=1$ or singlet state $S=0$. We refer to their singlet configurations in which they appear as bound states (generally below the energies of neutral excitons). The observation of triplet trions is more elusive. ${ }^{56-59}$ In the ESI $\dagger$, we evaluate experimental time resolved and integrated PL data (Fig. S3-S5 $\dagger$ ) for CdSe platelet samples of varying lateral size at low temperature $(T=4 \mathrm{~K})$. Using a numerical model, which accounts for population transfer between exciton and trion states under pulsed excitation we fit the experimental transients numerically (section S3 of the ESI $\dagger$ ). The model (Fig. 1(d)) takes four levels into consideration: the crystal ground state $|0\rangle$, the electron state |continuum $\rangle$ (from e-h continuum), the excitonic state $|X\rangle$, and trion state $\left|X^{-}\right\rangle$. We also compare the resultant power dependence of the time-integrated PL intensity from the rate equation model with the experimental findings in Fig. S5 $\uparrow$ and obtain also good agreement between the model and experiment. The electron for trion formation is assumed to originate from a residual background doping (see ESI section $\mathrm{S} 3 \mathrm{~A} \dagger$ ).

From the fits to the rate equation model (section S3 A of the ESI $\dagger$ ) we obtain three fit parameters, the exciton radiative rate $\Gamma_{\mathrm{X}}^{\mathrm{r}}$, the trion radiative rate $\Gamma_{\mathrm{X}^{-}}{ }^{\mathrm{r}}$, and scattering rate $\gamma_{\mathrm{X}^{-}}{ }^{0}$. Hence, for low $T$ the PL decay is a function of only these parameters for fits. Table 1 summarizes the fit results displayed in Fig. S4 (ESI $\dagger$ ) for different sizes.

At first we analyze the trends in Table 1 phenomenologically and then provide an in-depth theoretical modeling. With increasing lateral platelet size, the radiative rate of the exciton increases. This leads to measured exciton lifetimes $\left(\tau_{\mathrm{x}}^{\mathrm{r}}\right)$ in the range of tens of picoseconds $\left(37 \mathrm{ps}\right.$ for $29 \times 8 \mathrm{~nm}^{2}$, and $16 \mathrm{ps}$ for $41 \times 13 \mathrm{~nm}^{2}$, see also Fig. 1(c)). These are about two orders of magnitude shorter than those for spherical CdSe nanocrystals $^{60,61}$ and a consequence of the so-called giant oscillator strength (GOST) effect. ${ }^{62}$ The radiative rate of a $2 \mathrm{D}$ exciton is proportional to the ratio of the area of the exciton coherent in-plane motion to the exciton Bohr radius $a_{\mathrm{B}}$ squared. 6,13,62,63 First indications for such a behavior have been found. . $3,5,27,28,64$ For NPLs, much smaller than the wavelength and much larger than $a_{\mathrm{B}}$ (the weak and intermediate confinement regime), the exciton oscillator strength can be given by (see also further below for detailed calculations): ${ }^{13,57}$

$$
f_{\mathrm{X}}=\left|\Omega_{\mathrm{QP}}^{(R)}\right|^{2} \frac{L_{x} L_{y}}{a_{\mathrm{X}}{ }^{2}}
$$

Here $\Omega_{\mathrm{QP}}^{(\mathrm{R})}$ is the vacuum Rabi coupling and $a_{\mathrm{X}}$ is the exciton spatial extension, equal to the exciton Bohr radius $a_{\mathrm{B}}{ }^{13,31,57}$ The equation shows, that the exciton oscillator strength increases with the ratio of the NPL area to the effective Bohr radius. For large NPLs, $a_{\mathrm{B}}$ is equal to the two-dimensional limit $a_{\mathrm{B}}^{2 D} .^{13}$ Hence, for a fixed value of $a_{\mathrm{B}}$ for larger platelets, the oscillator strength should approximately increase $e . g$. by a factor of 2.28, with increasing lateral size from $232 \mathrm{~nm}^{2}(29 \times$ 
$\left.8 \mathrm{~nm}^{2}\right)$ NPLs to $533 \mathrm{~nm}^{2}\left(41 \times 13 \mathrm{~nm}^{2}\right)$, in good agreement with our experimental results from Table 1, where a ratio of 2.37 is found. Extrapolating the radiative exciton lifetime (Table 1) towards a (much) larger platelet area (Fig. 1(c)), few ps lifetimes can be achieved, ${ }^{13,28,64}$ being also comparable in order of magnitude with some TMDC materials (1 ps for $\mathrm{WS}_{2}{ }^{65,66}$ and $\sim 10 \mathrm{ps}$ for $\mathrm{MoSe}_{2}{ }^{67}$ ). This sets the CdSe NPLs among the fastest nano emitters.

Moreover, Table 1 shows that the exciton radiative rate is much greater than the trion rate, resulting in $\Gamma_{\mathrm{X}}^{\mathrm{r}} / \Gamma_{\mathrm{X}^{-}}{ }^{\mathrm{r}} \sim 5-14$. This is a general trend also observed in III-V, ${ }^{68} \mathrm{II}_{-\mathrm{VI}}{ }^{69}$ and TMDC $^{65,66}$ semiconductor quantum wells, where e.g. about 15 times longer trion emission time in TMDC materials is ascribed to a lowering of the oscillator strength due to a stronger localization. For CdSe quantum dots the trion lifetime is comparable to that of the exciton in the strong confinement regime ${ }^{70}$ while for large QDs it can be longer than that of the excitons. ${ }^{71}$ In $2 \mathrm{D}$ systems the trion lifetime can be comparable or longer than the exciton lifetime. ${ }^{72}$

Actually, in 2D systems, when a photon transforms into a correlated exciton, the photon wave vector $\boldsymbol{Q}_{\mathrm{p}}$ transforms into the exciton center-of-mass plane wave with the same in-plane momentum projection providing good matching. When a trion is formed, two plane waves - one for the free electron and the photon - transform into one plane wave, the trion center of mass motion. The electron $\left(\boldsymbol{K}_{\mathrm{e}}\right)$, originally delocalized over the sample volume $L^{D}$, with $D$ the dimensionality, ends up being localized in the trion (in a volume of the order of the trion volume $a_{\mathrm{X}^{-}}{ }^{\mathrm{D}}$ ), while the whole trion is delocalized again over the sample volume. The mentioned localization leads to a $\left(\frac{a_{\mathrm{X}^{-}}}{L}\right)^{\mathrm{D}}$ reduction of the trion oscillator strength, as seen by the trend in Table 1 for the associated radiative rate. For larger
NPLs in the weak/intermediate confinement regime $\left(L \geq a_{\mathrm{B}}\right)$, $\frac{f_{\mathrm{X}^{-}}}{f_{\mathrm{X}}} \approx \frac{a_{\mathrm{X}^{-}}{ }^{2}}{L_{x} L_{y}}$ is valid, with $a_{\mathrm{X}^{-}}$, the trion Bohr radius (calculated later in Fig. 2(d)). ${ }^{57}$ The trion oscillator strength $f_{\mathrm{X}^{-}}$(being calculated later numerically) is far smaller than the exciton oscillator strength $f_{\mathrm{X}}$ (as $L_{x, y} \gg a_{\mathrm{X}^{-}}$), except for very poor samples having coherence lengths or sample size $L$ of the order of the trion spatial extension, which we do not have. Hence, the trion radiative rate is much less than the exciton rate in Table 1 , with predictions in ref. 57, while in a strong confined quantum dot $\left(L_{x, y} \sim a_{\mathrm{x}^{-}}\right)$it is near to one, as discussed above. We also notice an LO-phonon bottleneck in the scattering rate $\gamma_{\mathrm{x}^{-}}{ }^{0}$, showing its maximum for platelets with energy spacing near to the $25 \mathrm{meV}$ (ref. 73) LO-phonon energy. ${ }^{5,18}$

While the rate equation model does not take into account some features such as an exciton fine structure, which plays a minor role (see ESI section $\mathrm{S} 3 \mathrm{C} \uparrow$ for detailed discussion) or higher excited states, ${ }^{51}$ it describes consistently the key features and trends of the size tunable trion and exciton properties. In-depth theoretical modeling is performed in the next section and compared with these results.

\section{Theory for trions and excitons}

The NPLs are modeled as quantum box like structures with a strong $z$-confinement to 4.5 ML (coordinates $z_{\mathrm{e}, \mathrm{h}}$ ) and variable lateral confinement $\left(L_{x}, L_{y}\right)$. Within the effective-mass and envelope-function approximation, the Schrödinger equation of a neutral exciton in a NPL reads

$$
\left(H_{\mathrm{e}}\left(z_{\mathrm{e}}\right)+H_{\mathrm{h}}\left(z_{\mathrm{h}}\right)+H_{\mathrm{X}}^{\mathrm{IP}}\left(\rho_{\mathrm{e}}, \rho_{\mathrm{h}}\right)\right) \Psi_{\mathrm{X}}\left(r_{\mathrm{e}}, r_{\mathrm{h}}\right)=E_{\mathrm{X}} \Psi_{\mathrm{X}}\left(r_{\mathrm{e}}, r_{\mathrm{h}}\right)
$$

where $\boldsymbol{r}_{i}=\left(\boldsymbol{\rho}_{i}, z_{i}\right)$. The total exciton wave function $\Psi_{\mathrm{X}}\left(r_{\mathrm{e}}, r_{\mathrm{h}}\right)$ is split into two parts: (i) the confinement wave functions $\phi_{\mathrm{e}}\left(z_{\mathrm{e}}\right)$,
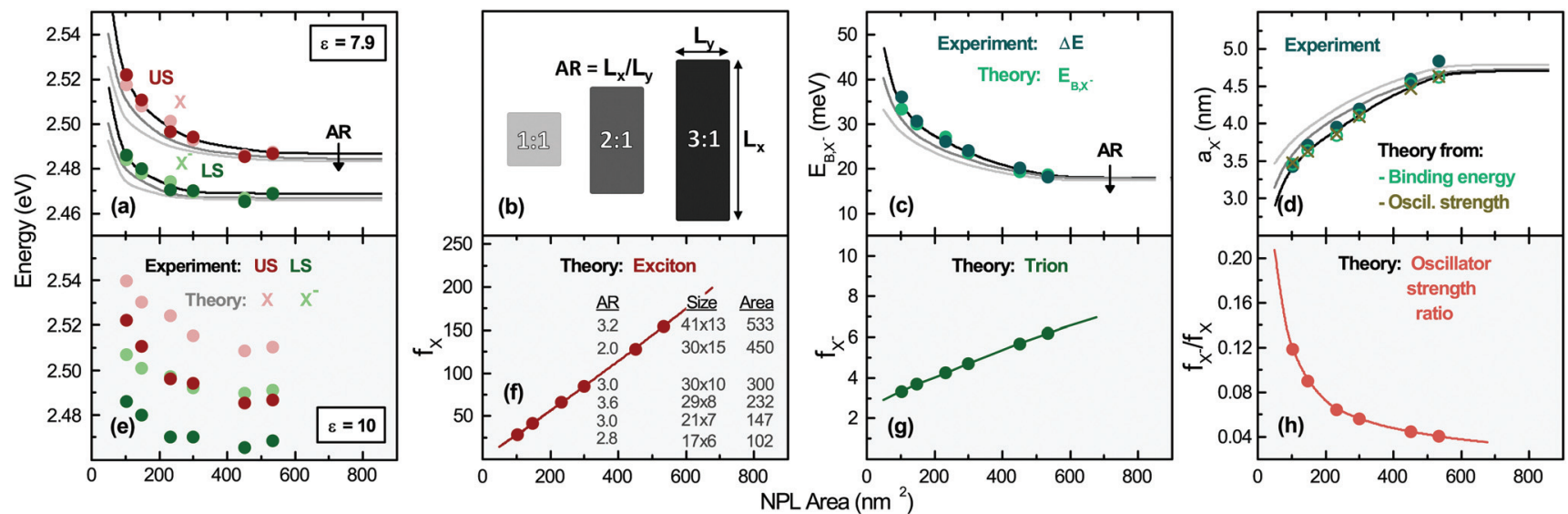

Fig. 2 (a) (US) and (LS) energies obtained from Fig. 1 (darker color data points) and theoretical model for $(X)$ and $\left(X^{-}\right)$, (pale color data points). Additionally the variation of exciton and trion energy with respect to the lateral NPL aspect ratio (AR) is shown, (lines of different brightness, see the legend in (b)). The energy of the trion and exciton is obtained by the numerical diagonalization of the matrix resulting from the projection of the Hamiltonian $\mathrm{H}_{\mathrm{X}}{ }^{-}$and $\mathrm{H}_{\mathrm{X}}$ for a fixed surrounding dielectric constant $\varepsilon_{\text {env }}=2.07$ and two different CdSe dielectric constants in (a) and (e). Partially single data points indicate that the data and model fall on top of each other. (b) Definition of the lateral aspect ratio, which varies from sample to sample, as given in (f). (c) Trion binding energy from the experiment and theory. The theoretical value dependence on the lateral NPL AR is also shown. (d) Trion Bohr radius from the experiment and theory. (f) Exciton and (g) trion oscillator strength and inverse oscillator strength ratio (h) for the samples. Different AR curves fall on top of each other. 
$\left(\phi_{\mathrm{h}}\left(z_{\mathrm{h}}\right)\right)$ of the lowest electron (heavy-hole) sublevel, which are solutions of the single electron (hole) Hamiltonian in the $z$-direction $H_{\mathrm{i}}\left(z_{\mathrm{i}}\right)=-\hbar^{2} / 2 m_{\perp \mathrm{i}}\left(\partial^{2} / \partial z_{\mathrm{i}}^{2}\right)+V_{\mathrm{i}}\left(z_{\mathrm{i}}\right)$. Here $V_{\mathrm{i}}\left(z_{\mathrm{i}}\right)$ is the sum of all possible single-particle potentials affecting particle $i$ $\left(\mathrm{i}=\mathrm{e}, \mathrm{h}\right.$ ), and $m_{\perp \mathrm{i}}$ is the mass of carrier $\mathrm{i}$ along the strongly confined $z$-direction ([001]), taken from ref. 37. (ii) The inplane wave function $\psi_{\mathrm{X}}^{\mathrm{IP}}\left(\rho_{\mathrm{e}}, \rho_{\mathrm{h}}\right)$ (index IP for in-plane) is the solution of the following in-plane Schrödinger equation:

$$
\begin{aligned}
& \left(\frac{\boldsymbol{p}_{\mathrm{e}}{ }^{2}}{2 m_{\mathrm{e} \|}}+\frac{\boldsymbol{p}_{\mathrm{h}}{ }^{2}}{2 m_{\mathrm{h} \|}}+V_{\mathrm{e}}\left(\boldsymbol{\rho}_{\mathrm{e}}\right)+V_{\mathrm{h}}\left(\boldsymbol{\rho}_{\mathrm{h}}\right)+\widehat{V}_{\mathrm{c}}\left(\boldsymbol{\rho}_{\mathrm{e}}-\boldsymbol{\rho}_{\mathrm{h}}\right)\right) \\
& \cdot \psi_{\mathrm{X}, \mathrm{j}}^{\mathrm{IP}}\left(\boldsymbol{\rho}_{\mathrm{e}}, \boldsymbol{\rho}_{\mathrm{h}}\right)=E_{\mathrm{X} 0, j}^{\mathrm{IP}} \psi_{\mathrm{X}, j}^{\mathrm{IP}}\left(\boldsymbol{\rho}_{\mathrm{e}}, \boldsymbol{\rho}_{\mathrm{h}}\right)
\end{aligned}
$$

where $\rho_{\mathrm{i}}$ are the in-plane electron $(\mathrm{i}=\mathrm{e})$ and hole $(\mathrm{i}=\mathrm{h})$ position vectors, $p_{\mathrm{i}}=-i \hbar \nabla \rho_{\mathrm{i}}$ is the in plane momentum operator, $m_{\mathrm{i} \| \mid}$ is the in-plane mass of carrier i used from ref. 37, and $V_{\mathrm{i}}\left(\boldsymbol{\rho}_{\mathrm{i}}\right)$ are the confinement potentials of the electron and hole (see Methods for details of the calculations). The model takes explicitly also the nonidentical lateral NPL length $\left(L_{x}, L_{y}\right)$ into account. It also treats screening using a Rytova-Keldysh potential, ${ }^{7,31,32,51,74-77}$ as the nanoplatelets are surrounded by oleic acid ligands, providing a dielectric contrast to the platelet core (see Methods).

For the negative trions within the effective-mass approximation, the Schrödinger equation can be written as: $\left(\sum_{\mathrm{i}=\mathrm{e}_{1}, \mathrm{e}_{2}, \mathrm{~h}} H_{\mathrm{i}}\left(z_{i}\right)+H_{\mathrm{X}^{-}}^{\mathrm{IP}}\right) \Psi_{\mathrm{X}^{-}}\left(r_{\mathrm{e} 1}, r_{\mathrm{e} 2}, r_{\mathrm{h}}\right)=E_{\mathrm{X}^{-}} \Psi_{\mathrm{X}^{-}}\left(r_{\mathrm{e} 1}, r_{\mathrm{e} 2}, r_{\mathrm{h}}\right)$. The total wave function of the trion solution of $H_{\mathrm{X}^{-}}$can be factorized into: $\Psi_{\mathrm{X}^{-}}\left(r_{\mathrm{e} 1}, r_{\mathrm{e} 2}, r_{\mathrm{h}}\right)=\phi_{\mathrm{e} 1}\left(z_{\mathrm{e} 1}\right) \phi_{\mathrm{e} 2}\left(z_{\mathrm{e} 2}\right) \phi_{\mathrm{h}}\left(z_{\mathrm{h}}\right) \psi_{\mathrm{X}^{-}, \mathrm{j}}{ }^{\mathrm{IP}}\left(\rho_{\mathrm{e} 1}, \rho_{\mathrm{e} 2}\right.$, $\left.\rho_{\mathrm{h}}\right)$, where $\phi_{\mathrm{i}}\left(z_{\mathrm{i}}\right)$ are the confinement functions of the lowest electron $\left(\mathrm{i}=\mathrm{e}_{1}, \mathrm{e}_{2}\right)$ and heavy-hole sublevel $(\mathrm{i}=\mathrm{h})$ and $\psi_{\mathrm{X}^{-}, \mathrm{j}}{ }^{\mathrm{IP}}\left(\rho_{\mathrm{e} 1}, \rho_{\mathrm{e} 2}, \rho_{\mathrm{h}}\right)$ is the wave function solution of the in-plane trion Hamiltonian:

$$
\begin{aligned}
& \left(\frac{\boldsymbol{p}_{\mathrm{e}_{1}}{ }^{2}}{2 m_{\mathrm{e} \|}}+\frac{\boldsymbol{p}_{\mathrm{e}_{2}}{ }^{2}}{2 m_{\mathrm{e} \|}}+\frac{\boldsymbol{p}_{\mathrm{h}}{ }^{2}}{2 m_{\mathrm{h} \|}}+V_{\mathrm{e}_{1}}\left(\boldsymbol{\rho}_{\mathrm{e}_{1}}\right)+V_{\mathrm{e}_{2}}\left(\boldsymbol{\rho}_{\mathrm{e}_{2}}\right)\right. \\
& \left.+V_{\mathrm{h}}\left(\boldsymbol{\rho}_{\mathrm{h}}\right)+\widehat{V}_{\mathrm{c}}\left(\boldsymbol{\rho}_{\mathrm{e}_{1}}-\boldsymbol{\rho}_{\mathrm{h}}\right)+\widehat{V}_{\mathrm{c}}\left(\boldsymbol{\rho}_{\mathrm{e}_{2}}-\boldsymbol{\rho}_{\mathrm{h}}\right)-\widehat{V}_{\mathrm{c}}\left(\boldsymbol{\rho}_{\mathrm{e}_{2}}-\boldsymbol{\rho}_{\mathrm{e}_{1}}\right)\right) \\
& \cdot \psi_{\mathrm{X}^{-}, j}^{P}\left(\rho_{\mathrm{e} 1}, \rho_{\mathrm{e} 2}, \rho_{\mathrm{h}}\right)=E_{\mathrm{X}^{-}, j}{ }^{I P} \psi_{\mathrm{X}^{-}, j}{ }^{I P}\left(\rho_{\mathrm{e} 1}, \rho_{\mathrm{e} 2}, \rho_{\mathrm{h}}\right)
\end{aligned}
$$

To investigate the negative trion binding energy of CdSe NPLs, we adopt the same formalism as for the exciton using the relative $\boldsymbol{\rho}_{\boldsymbol{i}}=\boldsymbol{\rho}_{\mathrm{ei}}-\boldsymbol{\rho}_{\mathrm{h}}$ and the center of mass $\boldsymbol{R}_{\mathrm{X}^{-}}=\left(m_{\mathrm{e}} \rho_{\mathrm{e} 1}+\right.$ $\left.m_{\mathrm{e}} \rho_{\mathrm{e} 2}+m_{\mathrm{h}} \rho_{\mathrm{h}}\right) / M_{\mathrm{X}^{-}}$trion coordinates. Hence the trion in-plane Hamiltonian is the sum of center of mass and relative Hamiltonian $\mathrm{H}_{\mathrm{X}^{-}}{ }^{\mathrm{IP}}=\mathrm{H}_{\mathrm{X}^{-}}{ }^{\mathrm{CM}}+\mathrm{H}_{\mathrm{X}^{-}}{ }^{\text {rel }}$, where the eigenenergy and eigenvector solutions of the in-plane Hamiltonian can be written as $\psi_{\mathrm{X}^{-}, j}^{\mathrm{IP}}\left(\boldsymbol{\rho}_{e_{1}}, \boldsymbol{\rho}_{e_{2}}, \boldsymbol{\rho}_{\boldsymbol{h}}\right)=\zeta_{\widetilde{1 S, N, M}}^{\mathrm{CM}}\left(R_{X^{-}}\right) \phi_{1 S, n l}^{\mathrm{rel}}\left(\rho_{1}, \rho_{2}\right)$ and

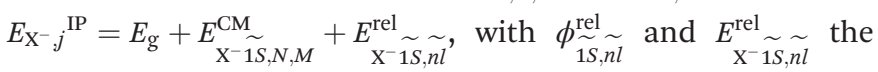
wave function and the energy solution of the relative Hamiltonian $H_{\mathrm{X}^{-}}{ }^{\text {rel }}$ given by:

$$
H_{\mathrm{X}^{-}}{ }^{\mathrm{rel}}=\sum_{i=1,2} H_{\mathrm{X}_{i}}{ }^{\mathrm{rel}}-\frac{\hbar^{2}}{m_{\mathrm{h}}} \nabla_{\rho_{1}} \cdot \nabla_{\rho_{2}}+\widehat{V}_{\mathrm{c}}\left(\left|\boldsymbol{\rho}_{1}-\boldsymbol{\rho}_{2}\right|\right)
$$

Here $H_{\mathrm{X}_{\mathrm{i}}}{ }^{\text {rel }}$ is the relative Hamiltonian of the neutral exciton and $M_{\mathrm{X}^{-}}=2 m_{\mathrm{e}}+m_{\mathrm{h}}$ is the trion mass. To solve the eigenvalue equation, we use a wave function expansion technique. (See Methods for further details.)

Fig. 2(a) reports the theory results of the exciton and trion energies for different platelet sizes. The experimental values (obtained from Fig. S3†) are also included for comparison, showing very good agreement. We can clearly notice that the exciton and trion energy for using the high-frequency CdSe dielectric constant $\left(\varepsilon_{\mathrm{CdSe}, \infty}=7.9\right)$ in the calculations for the treatment of screening provides a very good agreement, while the use of the static dielectric constant $\left(n_{\mathrm{CdSe}, \mathrm{s}}=10\right.$, Fig. $\left.2(\mathrm{e})\right)$ provides an overestimate of the transition energies, known in the literature and in line with trends in ref. 37. (See also Methods.) Further recent studies also suggest an effective dielectric constant of $6.4^{78}$ (averaging over the field components inside the platelet and outside in the ligands for the screened lowest exciton state). It has to be slightly lower than the inside dielectric constant we use, since the outside dielectric constant is near to two. Hence this further substantiates our $\varepsilon=7.9$ value.

Increasing the lateral platelet confinement results in a blue shift of about $\sim 18 \mathrm{meV}$ for the trion and $\sim 35 \mathrm{meV}$ for the exciton, while the lateral size is reduced from 533 to $102 \mathrm{~nm}^{2}$. (See also the Methods section for more details.) Theory curves for varying lateral aspect ratio (AR, see (b) for definition) are also displayed. We remark that our calculations are further substantiated as they result $e . g$. in an exciton binding energy of $201 \mathrm{meV}$ for $533 \mathrm{~nm}^{2}\left(41 \times 13 \mathrm{~nm}^{2}\right)$ platelets in good agreement with experiments in refs 28 and 39 . We will analyze the dependence of the binding energy and other parameters in Fig. 3.

In panel (c) of Fig. 2 the trion binding energy, corresponding to the energy separation between $X$ and $X^{-}$, is plotted vs. platelet area. It decreases from $33 \mathrm{meV}$ for $102 \mathrm{~nm}^{2}(17 \times$ $6 \mathrm{~nm}^{2}$ to $19 \mathrm{meV}$ for $\left.533 \mathrm{~nm}^{2}\left(41 \times 13 \mathrm{~nm}^{2}\right)\right)$ platelets. Going from laterally extended platelets to smaller ones, the calculated trion binding energies in our 2D system start with comparable values as e.g. for large quantum dots (14-22 meV) ${ }^{79,80}$ and get higher with decreased lateral size approaching the range of strong confined CdSe quantum dots (30-50 meV). ${ }^{71}$ A slight deviation for the smallest platelets considered $\left(102 \mathrm{~nm}^{2}, 17 \times\right.$ $6 \mathrm{~nm}^{2}$ ) is explained by the increasingly quantum dot like nature, while the model is exact for weak to intermediate lateral confinement. The continuous lines in Fig. 2(a) and (c), obtained from our calculations, show that the transition energies also depend on the lateral aspect ratio (AR).

In panel (f) and ( $\mathrm{g}$ ) of Fig. 2 we plot the calculated exciton and trion oscillator strength $f_{\mathrm{i}} \propto\left|\left\langle\Psi\left|\delta_{\text {re }}, r_{\mathrm{h}}\right| \Psi\right\rangle\right|^{2}$, see ESI section S1 B.† Both exciton and trion oscillator strengths increase with the NPL area, consistent with the theory of giant oscillator strength in quantum wells ${ }^{62}$ and weakly confined quantum dots. Theory curves for different AR values fall on top of each other. $f_{\mathrm{X}^{-}}$is far smaller than that of the exciton $\left(f_{\mathrm{X}-} / f_{\mathrm{X}}<1\right)$, consistent with our assumption of a more quantum well like than quantum dot like system for the lowest state. This is a result of the weaker localization of the trion compared to the exciton and in line with expectations based 

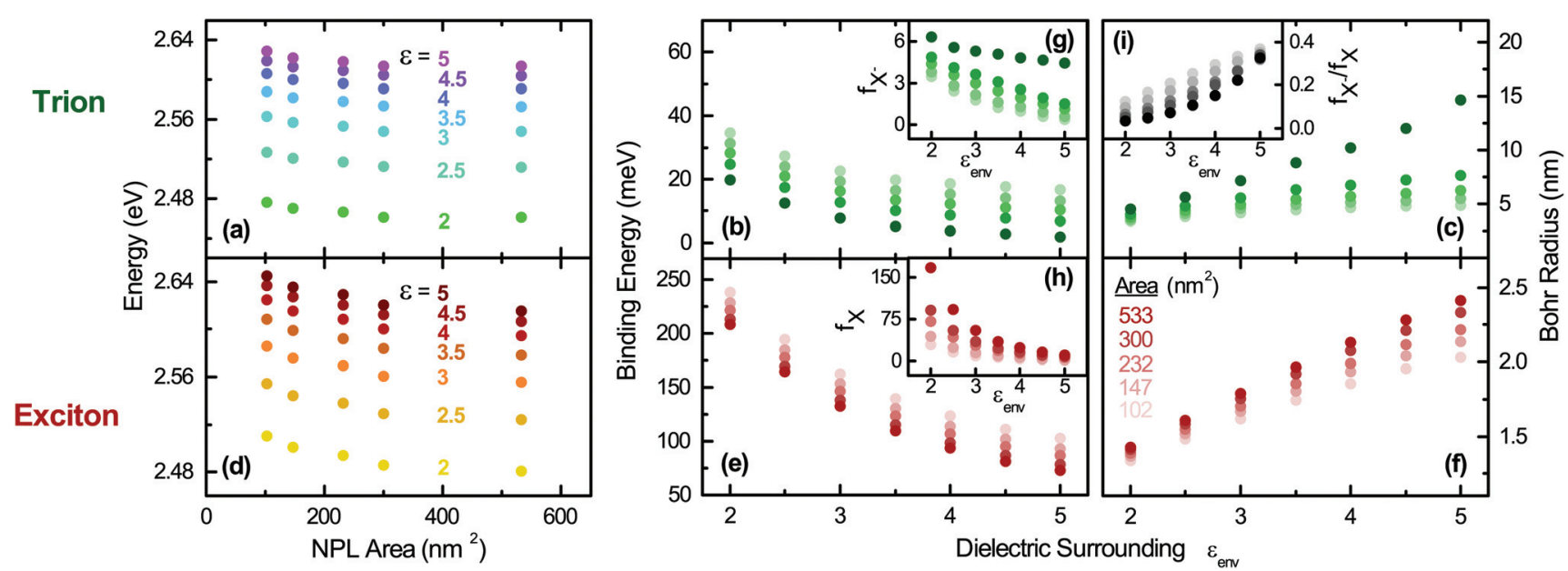

Fig. 3 Effect of the dielectric environment on the optical properties of the trions and excitons for different NPL sizes; Trion: (a) transition energy, (b) binding energy, and (c) Bohr radius. Exciton: (d) transition energy, (e) binding energy, and (f) Bohr radius. Insets: (g) Trion and (h) exciton oscillator strength as well as their ratio in (i). A high frequency dielectric constant $\varepsilon_{\mathrm{CdSe}, \infty}=7.9$ is always employed for the CdSe platelet core.

on Combescot et al. ${ }^{57}$ The variation of the oscillator strength of both species with the lateral size and the relative trion to exciton oscillator strength ratio (Fig. 2(h)) support our fitting parameters presented in Table 1 . The trion oscillator strength is an order of magnitude lower as for the excitons, also reflected in the experimental radiative rates in Table 1. In line with our results, much lower radiative rates of trions in quantum wells as compared to excitons have been predicted $^{56,81}$ at low temperatures and found in epitaxial II-VI and III-V wells. ${ }^{82}$ If the predicted oscillator strength of the exciton is translated to a radiative rate, ${ }^{13,26,83,84}$ we obtain 12 $\mathrm{ns}^{-1}$ to $62 \mathrm{~ns}^{-1}$ for the exciton transition. (See Table 1, Fig. 1(c); see also the ESI $\dagger$ for the method of calculation.) These values are in agreement with our experimental results validating the model. A slight deviation for the smallest sample is attributed to its increasingly quantum dot like nature.

Fig. 2(d) displays the relative motion extension of the trion $^{85}$ (Bohr radius) $a_{\mathrm{X}^{-}}=\sqrt{\hbar^{2} /\left(2 \mu_{\mathrm{X}^{-}} E_{\mathrm{B}^{-}}\right)}$, deduced from theory, as a function of the platelet area. As in (a), the specific sample lateral aspect ratio (given in (f)) is used for the calculations (data points), while the continuous lines represent the theory for different lateral aspect ratios. For the experimental data, the energy spacing in Table 1 is used with the formula above. $\mu_{\mathrm{X}^{-}}=M_{\mathrm{X}} m_{\mathrm{e}} /\left(M_{\mathrm{X}}+m_{\mathrm{e}}\right)$ is the trion relative motion mass. In order to validate our theoretical calculations, we plot in the same figure $a_{\mathrm{X}^{-}}$calculated using the oscillator strength ratio in (h): $a_{\mathrm{X}^{-}}=\sqrt{A \cdot\left(f_{\mathrm{X}^{-}} / f_{\mathrm{X}}\right)}$ ('Oscill. strength'). ${ }^{57,85,86}$ Further we deduce $a_{\mathrm{X}^{-}}$from the experimental results (trion-exciton energy difference) using the first mentioned equation and making use of ref. 85 and 86 . The trion extension $a_{\mathrm{X}^{-}}$calculated using our models is consistent with the experimental result, substantiating our model. Slight deviations occur for the $102 \mathrm{~nm}^{2},(17$ $\times 6 \mathrm{~nm}^{2}$ ) platelets, which are increasingly quantum dot like.

We remark that we decided to plot all trion and exciton properties in Fig. 2 versus the platelet area, as with varying lateral aspect ratio AR only the shortest length $\left(L_{y}\right)$ impacts the properties. A detailed discussion of the dependence of trion energy, binding energy and oscillator strength on the shortest platelet length $L_{y}$, which dominates the lateral confinement for platelets with higher lateral aspect ratios, is presented in section S1 C of the ESI. $\dagger$

In order to provide further insight into the optical properties of the CdSe NPLs, we investigate in Fig. 3 the effect of the dielectric environment on the transition energies, binding energies, Bohr radii and oscillator strength of trions and excitons by varying the surroundings (ligand) $\varepsilon_{\text {env }}$ and the platelet area. Therefore, the former parameter is varied in the calculations between $\varepsilon=\varepsilon_{\text {env }}=2$ and $\varepsilon=5$ to cover a large span of potential dielectric surroundings from strong dielectric contrast to weak dielectric contrast to the inside dielectric constant of 7.9 (as in Fig. 2(a)). As shown in Fig. 3, it is clear that the optical properties of the exciton and trion are sensitive to the dielectric environment and subsequently to the choice of the surrounding (e.g. organic ligands). E.g. for a fixed NPL size $\left(41 \times 13 \mathrm{~nm}^{2}\right.$ or $\left.533 \mathrm{~nm}^{2}\right)$ the absolute trion and exciton transition energies (Fig. 3(a) and (d) are only weakly altered. This is due to the decrease of the exciton and trion binding energy subtracted from the sum of the high bandgap $(1.766 \mathrm{eV}$ for CdSe) and confinement energy ( $\sim 0.9 \mathrm{eV})$. The exciton binding energy decreases by $135 \mathrm{meV}$ from $208 \mathrm{meV}$ to $73 \mathrm{meV}$ while $\varepsilon_{\text {env }}$ is increased from 2 to 5 , see Fig. 3(e). Our calculations result in an exciton binding energy of $201 \mathrm{meV}$ for $41 \times 13 \mathrm{~nm}^{2}$ platelets and $\varepsilon_{\mathrm{env}}=2.07$ (oleic acid), in good agreement with experimental determinations in ref. 28 and 39. The trion binding energy is more sensitive to the environment and drops from 20 to $2 \mathrm{meV}$ (Fig. 3(b)). However, most organic ligands (including oleic acid used here with $\varepsilon_{\mathrm{env}}=2.07$ ) have high frequency dielectric constants near to 2 , so that in an experiment only minor shifts would be observed upon ligand exchange. In contrast, a variation of the dielectric surrounding 
is possible using (colloidal) atomic layer deposition (ALD) methods ${ }^{87}$ for controlled deposition of a few monolayers of a high bandgap semiconductor or dielectric. In line with the trend in Fig. 3(e) the binding energy can be reduced considerably, e.g. upon CdS coating. ${ }^{9}$

Using our $\sim 200 \mathrm{meV}$ exciton binding energy and an about $\sim 20 \mathrm{meV}$ trion binding energy for the larger platelets we obtain an $E_{\mathrm{X}}^{\mathrm{B}}-/ E_{\mathrm{X}}^{\mathrm{B}}$ ratio of 0.1. This is in good agreement with predictions for trions in ideal quantum wells ${ }^{54,88,89}$ predicting a ratio of 0.12 . Hence, it further confirms our calculations. From our trion binding energy of the largest platelet we can calculate in Fig. 3(c) a trion Bohr radius of $4.6 \mathrm{~nm}$ (for $\varepsilon_{\text {env }}=$ 2.07) using the previously mentioned equation. It is in good agreement with the experimental value (Fig. 2(d)) calculated from the double peak energy spacing $(4.8 \mathrm{~nm})$. The corresponding exciton Bohr radius of $1.6 \mathrm{~nm}$ (compare Fig. 3(f)), calculated using $a_{\mathrm{X}}=\left(\hbar^{2} / 2 \mu E_{\mathrm{X}}^{\mathrm{B}}\right)^{1 / 2}$, is in line with the trend in Brumberg et al. ${ }^{39}$ having measured about 1.15 to $1.4 \mathrm{~nm}$, for smaller sizes (17-65 $\mathrm{nm}^{2}$ ). The Bohr radius increases in Fig. 3(f) with the lateral size. An increase of $\varepsilon_{\text {env }}$ leads to an increase of the Bohr radius ((c) and (f)) and a reduction of the spatial overlap between the electron and hole in their relative motion. This results in reduced oscillator strength $((\mathrm{g})$ and $(\mathrm{h}))$ for both trions and excitons.

Notably, our results in Fig. $3(\mathrm{~g})$ and (h) emphasize that in line with the binding energy, the negatively charged exciton oscillator strength is less sensitive to the dielectric environment ${ }^{5,18}$ compared to the exciton oscillator strength for a given lateral size. Both $f_{\mathrm{X}-}$ and $f_{\mathrm{X}}$ increase by trend with the lateral size of the NPLs (brightness coded area scale in (f)), in line with the trends shown in Table 1, where a fastening of the radiative rates is observed.

Transferring the exciton oscillator strength e.g. in a radiative rate (see section $\mathrm{S} 1 \mathrm{~B}$ of the ESI $\dagger$ ) results in good agreement with experiments. As the change of oscillator strength with increasing lateral platelet size is different for trions and excitons, this results in a reduction of the trion to exciton oscillator strength ratio with increasing lateral size shown in (i). For a fixed size, the ratio increases with $\varepsilon_{\text {env }}$ as $f_{\mathrm{X}}$ is more sensitive compared to $f_{\mathrm{X}^{-}}$, see $(\mathrm{g})$ and $(\mathrm{h})$. A further important aspect is that using low dielectric constant ligands or atomic layer deposition high dielectric constant coating the trion emission can be suppressed or enhanced with respect to the exciton emission and its oscillator strength. Furthermore, our results imply that the trion binding energy can be tuned strongly above and below the room temperature thermal energy (Fig. 3(b)), while the exciton binding energy (Fig. 3(f)) is always above the room temperature thermal energy. As a consequence, either no trions or a considerable amount is present $e . g$. in devices, where the former is desired to enhance the emission efficiency.

Summing up our theoretical results for the trion and exciton properties, they are consistent with our experimental results on CdSe platelet PL dynamics and time-integrated and power-dependent PL as well as the energy separation and state energies. Our results show that especially the trion binding energies, Bohr radii and oscillator strength can be tuned by the lateral size and dielectric environment. Nanoplatelets are thus a model system to study the tunability of trion properties in a laterally finite $2 \mathrm{D}$ system. The trion plus exciton model is also fully compatible with reported findings in the literature. (See section S5 of the ESI $\uparrow$ for a detailed discussion.)

\section{Conclusion}

In summary, we demonstrated that CdSe nanoplatelets are a model system to study the presence and tunability of trions and excitons in laterally finite $2 \mathrm{D}$ semiconductors. Based on theoretical modeling of transition energies, trion binding energies and oscillator strength of trions and excitons we showed that these properties can be tuned via the lateral size and aspect ratio. We further demonstrated that dielectric screening by the high frequency dielectric constant of the surroundings strongly changes e.g. the trion Bohr radius and oscillator strength. Using low dielectric constant ligands or atomic layer deposition high dielectric constant coating e.g. either trion emission can be suppressed or enhanced with respect to the exciton emission. Furthermore, our results imply that the trion binding energy can be tuned strongly above and below the room temperature thermal energy. Combining both effects, no trions or a considerable amount is present paving the way e.g. to an effective control of the emission efficiency of devices. Built on the 2D effective-mass approximation and solving the full four dimensional schrödinger equation for anisotropic platelets, we have investigated the lateral size tunability of the exciton (upper state) and trion (lower state) energies in CdSe NPLs including dielectric confinement. The trion binding energy becomes tunable and increases from $18 \mathrm{meV}$ to $36 \mathrm{meV}$ with lateral platelet quantization. The obtained size tunable trion Bohr radius $(\sim 3.5-4.8 \mathrm{~nm})$ is in excellent agreement with experiments. Using the exciton and trion wave function from modeling, we calculated the oscillator strengths of excitons and trions and demonstrated that the trion oscillator strength is far smaller and tunable. This seems to be a general trend, in line with strong confined conventional quantum dots. All these data are in good agreement and allow for our model to consistently describe the experimentally observed dependencies. Our theoretical model is further substantiated by the good agreement of the calculated very short radiative exciton lifetimes with the experimental results. The short lifetimes or high transition oscillator strength, being strongly tunable by the lateral size and lateral aspect ratio, set the nanoplatelets among the fastest nano emitters.

The presented results lay the foundation for further indepth studies of the photophysics of CdSe nanoplatelets and related structures (e.g. 2D perovskites, TMDCs), as the demonstrated exciton and trion manifold is expected to impact properties like gain mechanisms, lasing or transport strongly. Especially interesting is that systematic studies of the trion physics in a system with tunable, strongly anisotropic confinement become feasible. This e.g. opens up the possibility to 
investigate to which extent localization impacts trion formation and decay and relevant understanding also for MBE grown devices, where a finite coherence length or disorder potentials localize excitons and impact the detrimental trion formation, which limits the quantum yield and device performance for instance. Nanoplatelets are a system in-between the yet studied extrema of $0 \mathrm{D}$ quantum dots and laterally infinite quantum wells and allow strong tuning of the optical and dynamical properties, while the transition energies remain nearly constant. These unique properties feature nanoplatelets as an excellent model system to study trion and exciton properties.

\section{Methods}

\section{Theoretical simulations}

As discussed platelets have strong $z$-confinement (coordinates $z_{\mathrm{e}}$ and $z_{\mathrm{h}}$ ) and tunable lateral confinement. The Schrödinger equation of a neutral exciton in a NPL reads within the effective-mass and the envelope-function approximation: $\left(H_{\mathrm{e}}\left(z_{\mathrm{e}}\right)+H_{\mathrm{h}}\left(z_{\mathrm{h}}\right)+H_{\mathrm{X}}^{\mathrm{IP}}\left(\rho_{\mathrm{e}}, \rho_{\mathrm{h}}\right)\right) \Psi_{\mathrm{X}}\left(r_{\mathrm{e}}, r_{\mathrm{h}}\right)=E_{\mathrm{X}} \Psi_{\mathrm{X}}\left(r_{\mathrm{e}}, r_{\mathrm{h}}\right)$, where $\boldsymbol{r}_{\boldsymbol{i}}=$ $\left(\rho_{\mathrm{i}}, z_{\mathrm{i}}\right)$. The total exciton wave function $\Psi_{\mathrm{X}}\left(r_{\mathrm{e}}, r_{\mathrm{h}}\right)$ is split into two parts: (i) the confinement wave functions $\phi_{\mathrm{e}}\left(z_{\mathrm{e}}\right)\left(\phi_{\mathrm{h}}\left(z_{\mathrm{h}}\right)\right)$ of the lowest electron (heavy-hole) sublevel, which are solutions of the single electron (hole) Hamiltonian in the $z$ direction (perpendicular to the layers of the NPLs). $H_{i}\left(z_{i}\right)=-\hbar^{2} / 2 m_{\perp i}\left(\partial^{2} / \partial z_{i}^{2}\right)+V_{i}\left(z_{i}\right)$. Here $V_{\mathrm{i}}\left(z_{\mathrm{i}}\right)$ is the sum of all possible single-particle potentials affecting particle $(\mathrm{i}=\mathrm{e}, \mathrm{h})$, and $m_{\perp \mathrm{i}}$, is the mass of carrier $\mathrm{i}$ along the strongly confined $z$-direction ([001]), the latter are taken from ref. 37. (ii) The inplane wave function $\psi_{\mathrm{X}}^{\mathrm{IP}}\left(\rho_{\mathrm{e}}, \rho_{\mathrm{h}}\right)$ (index IP for in-plane) is the solution of the following in-plane Schrödinger equation:

$$
\begin{aligned}
& \left(\frac{\boldsymbol{p}_{\mathrm{e}}{ }^{2}}{2 m_{\mathrm{e} \|}}+\frac{\boldsymbol{p}_{\mathrm{h}}{ }^{2}}{2 m_{\mathrm{h} \|}}+V_{\mathrm{e}}\left(\boldsymbol{\rho}_{\mathrm{e}}\right)+V_{\mathrm{h}}\left(\boldsymbol{\rho}_{\mathrm{h}}\right)+\widehat{V}_{\mathrm{c}}\left(\boldsymbol{\rho}_{\mathrm{e}}-\boldsymbol{\rho}_{\mathrm{h}}\right)\right) \\
& \cdot \psi_{\mathrm{X}, j}^{\mathrm{P}}\left(\boldsymbol{\rho}_{\mathrm{e}}, \boldsymbol{\rho}_{\mathrm{h}}\right)=E_{\mathrm{X} 0, j}^{\mathrm{IP}} \psi_{\mathrm{X}, j}^{\mathrm{IP}}\left(\boldsymbol{\rho}_{\mathrm{e}}, \boldsymbol{\rho}_{\mathrm{h}}\right)
\end{aligned}
$$

It takes into account the defined platelet extent $\left(L_{x}, L_{y}\right)$ and aspect ratios of our samples. $\boldsymbol{\rho}_{\boldsymbol{i}}$ are the in plane electron $(\mathrm{i}=\mathrm{e})$, hole $(\mathrm{i}=\mathrm{h})$ position vectors, $p_{\mathrm{i}}=-i \hbar \nabla \rho_{\mathrm{i}}$ is the in plane momentum operator, $m_{\mathrm{i} \|}$ is the in-plane mass of carrier i used from ref. 37, and $V_{\mathrm{i}}\left(\rho_{\mathrm{i}}\right)$ are the confinement potentials of the electron and hole. NPLs are embedded within organic ligands having a relatively small dielectric constant $\left(\varepsilon_{\mathrm{env}}=2.07\right.$ for oleic acid ligands ${ }^{5}$ ).

Actually, the electric force lines emerging from charges within a semiconductor nanoparticle pass through the surrounding medium, having a smaller dielectric constant than the semiconductor. Therefore, in order to take the dielectric screening of coulomb interaction properly into account, the electron-hole direct Coulomb interaction is treated here using a Rytova-Keldysh potential $\widehat{V}_{\mathrm{c}}\left(\boldsymbol{\rho}_{\mathrm{e}}-\boldsymbol{\rho}_{\mathrm{h}}\right)=\frac{-e^{2}}{2 \pi \varepsilon_{\mathrm{env}} r_{\mathrm{s}}} \int^{\frac{\mathrm{e}^{i q .\left(\rho_{\mathrm{e}}-\rho_{\mathrm{h}}\right)}}{q\left(1+q r_{\mathrm{s}}\right)}} J_{0}\left(q\left|\boldsymbol{\rho}_{\mathrm{e}}-\boldsymbol{\rho}_{\mathrm{h}}\right|\right) d^{2} q$ according to the widely accepted approach. ${ }^{7,31,32,51,74-77}$ However to avoid the divergence of the integral of the Bessel function in the Keldysh potential $J_{0}\left(q\left|\rho_{\mathrm{e}}-\boldsymbol{\rho}_{\mathrm{h}}\right|\right)$, we can construct an approximate expression for $\widehat{V}_{\mathrm{c}}$ in terms of elementary functions $\widehat{V}_{\mathrm{c}}\left(\boldsymbol{\rho}_{\mathrm{e}}-\boldsymbol{\rho}_{\mathrm{h}}\right)=-\frac{e^{2}}{\varepsilon_{\mathrm{env}} r_{\mathrm{s}}}\left[\left(\frac{\left|\boldsymbol{\rho}_{\mathrm{e}}-\boldsymbol{\rho}_{\mathrm{h}}\right|}{\left|\boldsymbol{\rho}_{\mathrm{e}}-\boldsymbol{\rho}_{\mathrm{h}}\right|+r_{s}}\right)+(m a-\ln (2)) e^{\frac{\left|\boldsymbol{\rho}_{\mathrm{e}}-\rho_{\mathrm{h}}\right|}{r_{\mathrm{s}}}}\right]$ (see ref. 77 for details). This potential is better than using an unscreened vacuum Coulomb potential together with envelope functions in $z$-direction, the standard approach for quantum wells. Here, $r_{\mathrm{s}}=\varepsilon_{\mathrm{CdSe}} Z_{0} /\left(2 n_{\text {env }}\right)$ is the dielectric screening length, $Z_{0}=\left(a_{0} / 2\right) \times 4.5 \mathrm{ML}$ is the platelet thickness and $\gamma$ is the Euler constant.

CdSe NPLs can be in a weak (quantum well-like), intermediate or strong confinement regime (quantum dot-like), depending on their lateral size..$^{7,13,51}$ However, based on the recent results of ref. 7 and 51 the lowest energy exciton state can be described well by a weak confinement regime for the $x$ and $y$ directions, where the platelets have finite extent $L_{x}$ and $L_{y}$. Since larger platelets are in the focus of this paper $\left(L_{x}, L_{y}>a_{\mathrm{B}}^{3 \mathrm{D}}\right.$ $\sim 4 \mathrm{~nm}$, calculated using ref. 5) and we are interested in the lowest energy exciton state, the theoretical model for excitons will be built with the assumption of a weak (lateral) confinement regime, i.e. considering NPLs as a more quantum well like than quantum dot like structure. In this context, to target the weak confinement limit, we will at first (i) ignore the confinement potential and solve the Schrödinger equation including only Coulomb interaction besides the kinetic terms and (ii) use the center of mass approximation. In fact, by transforming $\boldsymbol{\rho}_{\mathrm{e}}$ and $\boldsymbol{\rho}_{\mathrm{h}}$ into $\boldsymbol{R}_{\mathrm{X}}=\frac{m_{e} \boldsymbol{\rho}_{\mathrm{e}}+m_{h} \boldsymbol{\rho}_{\mathrm{h}}}{M_{\mathrm{X}}}$ and $\boldsymbol{\rho}=\boldsymbol{\rho}_{\mathrm{e}}-\boldsymbol{\rho}_{\mathrm{h}}$, which are the position vector of the center of mass of the exciton and relative distance of the electron from the hole, respectively, the Hamiltonian in eqn (4) can be separated in center of mass $H_{\mathrm{X}}^{\mathrm{cm}}$ and relative motion $H_{\mathrm{X}}^{\text {rel }}$ Hamiltonians, which are solved numerically using the finite lateral size $\left(L_{x}\right.$, $L_{y}$ ) of the NPLs.

$M_{\mathrm{X}}=m_{\mathrm{e}}+m_{\mathrm{h}}$ is the exciton mass. The solutions of the resulting Schrödinger equation of the system satisfy the eigenequation $\left(H_{\mathrm{X}}^{\mathrm{CM}}+H_{\mathrm{X}}^{\mathrm{rel}}\right) \psi_{\mathrm{X}, \mathrm{j}}^{\mathrm{IP}}\left(R_{\mathrm{X}}, \rho\right)=E_{\mathrm{X}, \mathrm{j}}^{\mathrm{IP}} \psi_{\mathrm{X}, \mathrm{j}}^{\mathrm{IP}}\left(R_{\mathrm{X}}, \rho\right)$ and are given by $\chi_{\mathrm{X}, j}^{\mathrm{IP}}\left(R_{\mathrm{X}}, \rho\right)=\chi_{1 S, N, M}^{\mathrm{CM}}\left(R_{\mathrm{X}}\right) \xi_{\tilde{n}, \tilde{l}}(\rho, \theta), E_{\mathrm{X}, j}^{\mathrm{IP}}=E_{\tilde{n}, \tilde{l}}^{\mathrm{rel}}+E_{\widetilde{1 S, N, M}}^{\mathrm{CM}}+E_{\mathrm{g}}$. Here, $E_{\tilde{\mathrm{n}}, l}$ and $\xi_{\tilde{n}, l}(\rho, \theta)$ are the eigenenergy and the eigenvalue, respectively, obtained by numerical diagonalization of the relative Hamiltonian. $\boldsymbol{\xi}_{\tilde{n}, \imath}(\rho)$ can be expanded using an auxiliary basis along with $\xi_{\tilde{n}, \tilde{l}}(\rho, \theta)=\sum_{n, l} C(n, l) \varphi_{n, l}(\rho, \theta)$, where $\varphi_{\mathrm{n}, 1}(\rho, \theta)$ are the wave functions of the 2D-hydrogenic states given in terms of orthogonal associated Laguerre polynomials. $E_{\mathrm{X}, \widetilde{1 S}, N, M}^{\mathrm{CM}}$ and $\underset{1 S, N, M}{\mathrm{CM}}\left(R_{\mathrm{X}}\right)$ are the solution of the center of mass Hamiltonian, obtained by multiplying eqn (4) by $\xi_{\tilde{n}, \tilde{l}}^{*}(\rho, \theta)$ and integrating the full Schrödinger equation over $\rho$. (For more details see e.g. ref. 7 and 51). $E_{\mathrm{g}}$ is the energy gap between conduction and valence bands at the $\Gamma$ point, taken from ref. 90.

Excitons can form charged excitons (trions) with excess charges. For negative trions the Schrödinger equation can be written as: $\left(\sum_{\mathrm{i}=\mathrm{e}_{1}, \mathrm{e}_{2}, \mathrm{~h}} H_{i}\left(z_{i}\right)+H_{\mathrm{X}^{-}}^{\mathrm{IP}}\right) \Psi_{\mathrm{X}^{-}}\left(r_{\mathrm{e} 1}, r_{\mathrm{e} 2}, r_{\mathrm{h}}\right)=E_{\mathrm{X}^{-}} \Psi_{\mathrm{X}^{-}}\left(r_{\mathrm{e} 1}, r_{\mathrm{e} 2}, r_{\mathrm{h}}\right)$. The 
total trion wave function of the Hamitonian $H_{\mathrm{X}^{-}}$can be factorized into: $\Psi_{\mathrm{X}-}\left(r_{\mathrm{e} 1}, r_{\mathrm{e} 2}, r_{\mathrm{h}}\right)=\phi_{\mathrm{e} 1}\left(z_{\mathrm{e} 1}\right) \phi_{\mathrm{e} 2}\left(z_{\mathrm{e} 2}\right) \phi_{\mathrm{h}}\left(z_{\mathrm{h}}\right) \psi_{\mathrm{X}^{-}, \mathrm{j}_{\mathrm{j}}}{ }^{\mathrm{IP}}\left(\rho_{\mathrm{e} 1}, \rho_{\mathrm{e} 2}\right.$, $\left.\rho_{\mathrm{h}}\right)$, where $\phi_{\mathrm{i}}\left(z_{\mathrm{i}}\right)$ are the confinement functions of the lowest electron ( $\left.\mathrm{i}=e_{1}, e_{2}\right)$ and heavy-hole sublevel $(\mathrm{i}=\mathrm{h})$, respectively, and $\psi_{\mathrm{X}^{-}, \mathrm{j}}{ }^{\mathrm{IP}}\left(\rho_{\mathrm{e} 1}, \rho_{\mathrm{e} 2}, \rho_{\mathrm{h}}\right)$ is the wave function solution of the in plane trion Hamiltonian:

$$
\begin{aligned}
& \left(\frac{\boldsymbol{p}_{\mathrm{e}_{1}}{ }^{2}}{2 m_{\mathrm{e} \|}}+\frac{\boldsymbol{p}_{\mathrm{e}_{2}}^{2}}{2 m_{\mathrm{e} \|}}+\frac{\boldsymbol{p}_{\mathrm{h}}{ }^{2}}{2 m_{\mathrm{h} \|}}+V_{\mathrm{e}_{1}}\left(\boldsymbol{\rho}_{\mathrm{e}_{1}}\right)+V_{\mathrm{e}_{2}}\left(\boldsymbol{\rho}_{\mathrm{e}_{2}}\right)\right. \\
& \left.+V_{\mathrm{h}}\left(\boldsymbol{\rho}_{\mathrm{h}}\right)+\widehat{V}_{\mathrm{c}}\left(\boldsymbol{\rho}_{\mathrm{e}_{1}}-\boldsymbol{\rho}_{\mathrm{h}}\right)+\widehat{V}_{\mathrm{c}}\left(\boldsymbol{\rho}_{\mathrm{e}_{2}}-\boldsymbol{\rho}_{\mathrm{h}}\right)-\widehat{V}_{\mathrm{c}}\left(\boldsymbol{\rho}_{\mathrm{e}_{2}}-\boldsymbol{\rho}_{\mathrm{e}_{1}}\right)\right) \\
& \cdot \psi_{\mathrm{X}^{-}, j}{ }^{\mathrm{IP}}\left(\boldsymbol{\rho}_{\mathrm{e} 1}, \boldsymbol{\rho}_{\mathrm{e} 2}, \boldsymbol{\rho}_{\mathrm{h}}\right)=E_{\mathrm{X}^{-}, j}{ }^{\mathrm{IP}} \psi_{\mathrm{X}^{-}, j}{ }^{\mathrm{IP}}\left(\boldsymbol{\rho}_{\mathrm{e} 1}, \boldsymbol{\rho}_{\mathrm{e} 2}, \boldsymbol{\rho}_{\mathrm{h}}\right)
\end{aligned}
$$

To investigate the negative trion binding energy in CdSe NPLs, we adopt the same formalism as for the exciton i.e. neglecting the confinement potential and using the relative $\boldsymbol{\rho}_{\mathrm{i}}$ $=\boldsymbol{\rho}_{\mathrm{ei}}-\boldsymbol{\rho}_{\mathbf{b}}$ and the center of mass $\boldsymbol{R}_{\mathrm{X}^{-}}=\frac{m_{\mathrm{e}} \boldsymbol{\rho}_{\mathrm{e}_{1}}+m_{\mathrm{e}} \boldsymbol{\rho}_{\mathrm{e}_{2}}+m_{\mathrm{h}} \boldsymbol{\rho}_{\mathrm{h}}}{M_{\mathrm{X}^{-}}}$ trion coordinates. Hence the trion in-plane Hamiltonian can be rewritten as a sum of center of mass and relative Hamiltonians $H_{\mathrm{X}^{-}}{ }^{\mathrm{IP}}=\mathrm{H}_{\mathrm{X}^{-}}{ }^{\mathrm{CM}}+{H_{\mathrm{X}^{-}}}^{\text {rel }}$, where the eigen energy and eigen vector solution of the in plane Hamiltonian can be rewritten as $\psi_{\mathrm{X}^{-}, j}^{\mathrm{IP}}\left(\rho_{e_{1}}, \rho_{e_{2}}, \rho_{\mathrm{h}}\right)=\zeta_{1 S, N, M}^{\mathrm{CM}}\left(R_{\mathrm{X}^{-}}\right) \phi_{1 S, n l}^{\mathrm{rrl}} \underset{\sim}{\mathrm{r}}\left(\rho_{1}, \rho_{2}\right)$ and

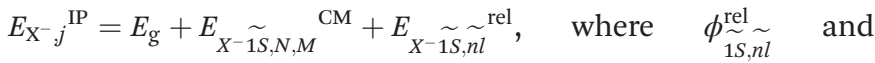
$E_{\mathrm{X}^{-} \tilde{T}, \tilde{n l}}^{\sim} \underset{\mathrm{rel}}{\sim}$ are the wave function and the energy solution of the relative Hamiltonian $H_{\mathrm{X}^{-}}{ }^{\text {rel }}$ given by $H_{\mathrm{X}^{-}}{ }^{\mathrm{rel}}=\sum_{i=1,2} H_{X_{i}}^{\mathrm{rel}}-\frac{\hbar^{2}}{m_{h}} \nabla_{\rho_{1}} \cdot \nabla_{\rho_{2}}+\hat{V}_{c}\left(\left|\boldsymbol{\rho}_{1}-\boldsymbol{\rho}_{2}\right|\right)$. Here $H_{\mathrm{Xi}}^{\mathrm{rel}}$ is the relative Hamiltonian of the neutral exciton and $M_{\mathrm{X}^{-}}=2 m_{\mathrm{e}}+m_{\mathrm{h}}$ is the trion mass.

To solve the eigenvalue equation, we use a wave function expansion technique. It is factorized into $\phi_{\widetilde{1 S}, \tilde{n l}}^{\mathrm{rel}}\left(\rho_{1}, \rho_{2}\right)=\sum_{\tilde{n} \tilde{l}} D(\tilde{n}, \tilde{l}) \frac{1}{\sqrt{2}}\left\{\xi_{\widetilde{\sim}}\left(\rho_{1}, \theta\right) \xi_{\widetilde{n l}}\left(\rho_{2}, \theta\right)+\xi_{\sim \mathcal{}}\left(\rho_{2}, \theta\right) \xi_{\tilde{n l}}\left(\rho_{1}, \theta\right)\right\}$, where $\xi_{n l}\left(\rho_{i}, \theta\right)=\sum_{n, l} C(n, l) \varphi_{n, l}(\rho, \theta)$ is the eigenvalue solution of the exciton Hamiltonian $H_{\mathrm{Xi}}^{\mathrm{rel}}$ (see ESI section $\mathrm{S} 1 \mathrm{~A} \uparrow$ for more details on the choice of relative basis functions). As for the exciton, the center of mass eigenvalue and eigenenergy are obtained by multiplying eqn (3) with $\phi_{1 S, n l}^{\text {rel }} \sim\left(\rho_{1}, \rho_{2}\right)$ and integrating the full Schrödinger eqn (3) over $\rho_{1}$ and $\rho_{2}$. Table 2 reports the used material constants applied in the theoretical simulations.

Table 2 CdSe material parameters used for the calculations

\begin{tabular}{lll}
\hline & CdSe parameters & Ref. \\
\hline$a_{0}(\mathrm{~nm})$ & 0.6052 & 91 \\
$E_{\mathrm{g}}(\mathrm{eV})$ & 1.766 & 90 \\
$E_{\mathrm{p}}(\mathrm{eV})$ & 16.5 & 3 \\
$\varepsilon_{\mathrm{CdSe}, \mathrm{s}}$ & 10 & 37 \\
$\varepsilon_{\mathrm{CdSe}, \infty}$ & 7.9 & 5 \\
$m_{\mathrm{e}} / m_{0}$ & 0.12 & 92 \\
$\gamma_{1}$ & 4.243 & 37 \\
$\gamma_{2}$ & 1.415 & 37 \\
$\gamma_{3}$ & 1.801 & 37
\end{tabular}

A comment on surrounding dielectric constant's high and low frequency values: in panel (e) of Fig. 2 we plot the exciton and trion energies for the static dielectric constant of CdSe NPLs $\varepsilon_{\mathrm{CdSe}, \mathrm{s}}=10$. We notice that the use of $\varepsilon_{\mathrm{CdSe}, \mathrm{s}}=10$ instead of $\varepsilon_{\mathrm{CdSe}, \infty}=7.9$ (panel (a)) would increase $E_{\mathrm{X}}$ and $E_{\mathrm{X}^{-}}$significantly. In the case of the exciton the calculated binding energies ( $200 \mathrm{meV}$, see the discussion in the main text) are much larger than the optical phonon energies in CdSe (25 meV). This implies that a high frequency dielectric constant should be used to calculate the NPL exciton ground state. However, in the case of the trion and for a sufficiently large NPL area (e.g. $\left.41 \times 13 \mathrm{~nm}^{2}\left(533 \mathrm{~nm}^{2}\right)\right)$, the binding energy of the trion $(\sim 18 \mathrm{meV})$ is smaller than the optical phonon energy. Hence, choosing between the static and high-frequency dielectric constants is a subtle problem when one wishes to calculate the trion states in CdSe NPLs. In our case, since the high dielectric constant gives a very good agreement with the experimental finding for both states (trion and exciton) we employ the highfrequency dielectric constant of CdSe. We should note that the energy shift (trion binding energy) is completely independent of these parameters. This can be reasoned directly from Fig. 2, e.g. for $21 \times 7 \mathrm{~nm}^{2}\left(147 \mathrm{~nm}^{2}\right)$ and $30 \times 15 \mathrm{~nm}^{2}\left(450 \mathrm{~nm}^{2}\right)$ platelets, where for the latter the trion binding energy is $29 \mathrm{meV}$ for both dielectric constants, while it is 19.6 and $19.2 \mathrm{meV}$ for the two different dielectric constants and $30 \times 15 \mathrm{~nm}^{2}$ platelets.

\section{Author contributions}

S.A., A.W.A., and S.J. developed and performed modeling and calculations. A.W.A. designed and performed measurements. S.C., G.H.V.B., I.M., and M.A. provided samples and conducted their characterization. N.O. and U.W. contributed to the discussion. A.W.A., S.A., and M.T.Q. wrote the manuscript.

\section{Conflicts of interest}

There are no conflicts to declare.

\section{Acknowledgements}

A. W. A. acknowledges funding by DFG projects AC290/2-1 and AC290/2-2 and I. M. by the European Research Council (ERC) Horizon 2020 research and innovation programme (grant no. 714876 PHOCONA).

\section{References}

1 S. Ithurria and B. Dubertret, J. Am. Chem. Soc., 2008, 130, 16504-16505.

2 A. W. Achtstein, A. Schliwa, A. Prudnikau, M. Hardzei, M. V. Artemyev, C. Thomsen and U. Woggon, Nano Lett., 2012, 12, 3151-3157. 
3 S. Ithurria, M. D. Tessier, B. Mahler, R. P. S. M. Lobo, B. Dubertret and A. L. Efros, Nat. Mater., 2011, 10, 936-941.

4 G. H. V. Bertrand, A. Polovitsyn, S. Christodoulou, A. H. Khan and I. Moreels, Chem. Commun., 2016, 52, 11975-11978.

5 A. W. Achtstein, R. Scott, S. Kickhöfel, S. T. Jagsch, S. Christodoulou, G. H. Bertrand, A. V. Prudnikau, A. Antanovich, M. Artemyev, I. Moreels, A. Schliwa and U. Woggon, Phys. Rev. Lett., 2016, 116, 116802.

6 J. Planelles, A. W. Achtstein, R. Scott, N. Owschimikow, U. Woggon and J. I. Climente, ACS Photonics, 2018, 5, 36803688.

7 M. Richter, Phys. Rev. Mater., 2017, 1, 016001.

8 D. P. Morgan, C. J. A. Maddux and D. F. Kelley, J. Phys. Chem. C, 2018, 122, 23772-23779.

9 A. W. Achtstein, O. Marquardt, R. Scott, M. Ibrahim, T. Riedl, A. V. Prudnikau, A. Antanovich, N. Owschimikow, J. K. N. Lindner, M. Artemyev and U. Woggon, ACS Nano, 2018, 12, 9476-9483.

10 B. Mahler, B. Nadal, C. Bouet, G. Patriarche and B. Dubertret, J. Am. Chem. Soc., 2012, 134, 18591-18598.

11 M. Nasilowski, P. Spinicelli, G. Patriarche and B. Dubertret, Nano Lett., 2015, 15, 3953-3958.

12 V. Dzhagan, A. G. Milekhin, M. Y. Valakh, S. Pedetti, M. Tessier, B. Dubertret and D. R. T. Zahn, Nanoscale, 2016, 8, 17204-17212.

13 F. Rajadell, J. I. Climente and J. Planelles, Phys. Rev. B, 2017, 96, 035307.

14 M. D. Tessier, B. Mahler, B. Nadal, H. Heuclin, S. Pedetti and B. Dubertret, Nano Lett., 2013, 13, 3321-3328.

15 E. V. Shornikova, L. Biadala, D. R. Yakovlev, D. Feng, V. F. Sapega, N. Flipo, A. A. Golovatenko, M. A. Semina, A. V. Rodina, A. A. Mitioglu, M. V. Ballottin, P. C. M. Christianen, Y. G. Kusrayev, M. Nasilowski, B. Dubertret and M. Bayer, Nano Lett., 2018, 18, 373-380.

16 L. Biadala, F. Liu, M. D. Tessier, D. R. Yakovlev, B. Dubertret and M. Bayer, Nano Lett., 2014, 14, 11341139.

17 E. V. Shornikova, L. Biadala, D. R. Yakovlev, V. F. Sapega, Y. G. Kusrayev, A. A. Mitioglu, M. V. Ballottin, P. C. M. Christianen, V. V. Belykh, M. V. Kochiev, N. N. Sibeldin, A. A. Golovatenko, A. V. Rodina, N. A. Gippius, A. Kuntzmann, Y. Jiang, M. Nasilowski, B. Dubertret and M. Bayer, Nanoscale, 2018, 10, 646-656.

18 R. Scott, A. V. Prudnikau, A. Antanovich, S. Christodoulou, T. Riedl, G. H. V. Bertrand, N. Owschimikow, J. K. N. Lindner, Z. Hens, I. Moreels, M. Artemyev, U. Woggon and A. W. Achtstein, Nanoscale, 2019, 11, 39583967.

19 C. J. A. Maddux, D. F. Kelley and A. M. Kelley, J. Phys. Chem. C, 2018, 122, 27100-27106.

20 R. Scott, A. W. Achtstein, A. Prudnikau, A. Antanovich, S. Christodoulou, I. Moreels, M. Artemyev and U. Woggon, Nano Lett., 2015, 15, 4985-4992.

21 J. Heckmann, R. Scott, A. V. Prudnikau, A. Antanovich, N. Owschimikow, M. Artemyev, J. I. Climente, U. Woggon,
N. B. Grosse and A. W. Achtstein, Nano Lett., 2017, 17, 6321-6329.

22 C. She, I. Fedin, D. S. Dolzhnikov, A. Demortière, R. D. Schaller, M. Pelton and D. V. Talapin, Nano Lett., 2014, 14, 2772-2777.

23 J. Q. Grim, S. Christodoulou, F. Di Stasio, R. Krahne, R. Cingolani, L. Manna and I. Moreels, Nat. Nanotechnol., 2014, 9, 891-895.

24 M. T. Quick, N. Owschimikow, A. H. Khan, A. Polovitsyn, I. Moreels, U. Woggon and A. W. Achtstein, Nanoscale, 2019, 11, 17293-17300.

25 Q. A. Akkerman, G. Rainò, M. V. Kovalenko and L. Manna, Nat. Mater., 2018, 17, 394-405.

26 A. W. Achtstein, A. Antanovich, A. Prudnikau, R. Scott, U. Woggon and M. Artemyev, J. Phys. Chem. C, 2015, 119, 20156-20161.

27 L. T. Kunneman, M. D. Tessier, H. Heuclin, B. Dubertret, Y. V. Aulin, F. C. Grozema, J. M. Schins and L. D. A. Siebbeles, J. Phys. Chem. Lett., 2013, 4, 3574-3578.

28 A. Naeem, F. Masia, S. Christodoulou, I. Moreels, P. Borri and W. Langbein, Phys. Rev. B: Condens. Matter Mater. Phys., 2015, 91, 121302.

29 R. Scott, J. Heckmann, A. V. Prudnikau, A. Antanovich, A. Mikhailov, N. Owschimikow, M. Artemyev, J. I. Climente, U. Woggon, N. B. Grosse and A. W. Achtstein, Nat. Nanotechnol., 2017, 17, 1155-1160.

30 D.-E. Yoon, W. D. Kim, D. Kim, D. Lee, S. Koh, W. K. Bae and D. C. Lee, J. Phys. Chem. C, 2017, 121, 24837-24844.

31 S. Ayari, A. Smiri, A. Hichri, S. Jaziri and T. Amand, Phys. Rev. B, 2018, 98, 205430.

32 A. Hichri, I. B. Amara, S. Ayari and S. Jaziri, J. Phys.: Condens. Matter, 2017, 29, 435305.

33 L. Waldecker, A. Raja, M. Rösner, C. Steinke, A. Bostwick, R. J. Koch, C. Jozwiak, T. Taniguchi, K. Watanabe, E. Rotenberg, T. O. Wehling and T. F. Heinz, Phys. Rev. Lett., 2019, 123, 206403.

34 V. A. Hintermayr, L. Polavarapu, A. S. Urban and J. Feldmann, ACS Nano, 2018, 12, 10151-10158.

35 Q. Wang, X.-D. Liu, Y.-H. Qiu, K. Chen, L. Zhou and Q.-Q. Wang, AIP Adv., 2018, 8, 025108.

36 J. A. Sichert, Y. Tong, N. Mutz, M. Vollmer, S. Fischer, K. Z. Milowska, R. García Cortadella, B. Nickel, C. Cardenas-Daw, J. K. Stolarczyk, A. S. Urban and J. Feldmann, Nano Lett., 2015, 15, 6521-6527.

37 R. Benchamekh, N. A. Gippius, J. Even, M. Nestoklon, J.-M. Jancu, S. Ithurria, B. Dubertret, A. L. Efros and P. Voisin, Phys. Rev. B: Condens. Matter Mater. Phys., 2014, 89, 035307.

38 R. Scott, A. W. Achtstein, A. V. Prudnikau, A. Antanovich, L. D. A. Siebbeles, M. Artemyev and U. Woggon, Nano Lett., 2016, 16, 6576-6583.

39 A. Brumberg, S. M. Harvey, J. P. Philbin, B. T. Diroll, B. Lee, S. A. Crooker, M. R. Wasielewski, E. Rabani and R. D. Schaller, ACS Nano, 2019, 13, 8589-8596.

40 T. C. Berkelbach, M. S. Hybertsen and D. R. Reichman, Phys. Rev. B: Condens. Matter Mater. Phys., 2013, 88, 045318. 
41 K. F. Mak, K. He, C. Lee, G. H. Lee, J. Hone, T. F. Heinz and J. Shan, Nat. Mater., 2013, 12, 207-211.

42 B. Ganchev, N. Drummond, I. Aleiner and V. Fal'ko, Phys. Rev. Lett., 2015, 114, 107401.

43 G. Plechinger, P. Nagler, A. Arora, R. Schmidt, A. Chernikov, A. G. del Águila, P. C. M. Christianen, R. Bratschitsch, C. Schüller and T. Korn, Nat. Commun., 2016, 7, 12715.

44 S. Helmrich, R. Schneider, A. W. Achtstein, A. Arora, B. Herzog, S. M. de Vasconcellos, M. Kolarczik, O. Schöps, R. Bratschitsch, U. Woggon and N. Owschimikow, 2D Mater., 2018, 5, 045007.

45 A. Arora, T. Deilmann, T. Reichenauer, J. Kern, S. Michaelis de Vasconcellos, M. Rohlfing and R. Bratschitsch, Phys. Rev. Lett., 2019, 123, 167401.

46 F. Cadiz, S. Tricard, M. Gay, D. Lagarde, G. Wang, C. Robert, P. Renucci, B. Urbaszek and X. Marie, Appl. Phys. Lett., 2016, 108, 251106.

47 A. Singh, G. Moody, K. Tran, M. E. Scott, V. Overbeck, G. Berghäuser, J. Schaibley, E. J. Seifert, D. Pleskot, N. M. Gabor, J. Yan, D. G. Mandrus, M. Richter, E. Malic, X. Xu and X. Li, Phys. Rev. B, 2016, 93, 041401.

48 K. Hao, L. Xu, P. Nagler, A. Singh, K. Tran, C. K. Dass, C. Schüller, T. Korn, X. Li and G. Moody, Nano Lett., 2016, 16, 5109-5113.

49 F. Gerdes, C. Navío, B. H. Juárez and C. Klinke, Nano Lett., 2017, 17, 4165-4171.

50 S. Christodoulou, J. I. Climente, J. Planelles, R. Brescia, M. Prato, B. Martín-Garcí, A. H. Khan and I. Moreels, Nano Lett., 2018, 18, 6248-6254.

51 J. F. Specht, R. Scott, M. C. Castro, S. Christodoulou, G. H. Bertrand, A. Prudnikau, A. Antanovich, L. Siebbeles, N. Owschimikow, I. Moreels, et al., Nanoscale, 2019, 11, 12230-12241.

52 B. T. Diroll, W. Cho, I. Coropceanu, S. M. Harvey, A. Brumberg, N. Holtgrewe, S. A. Crooker, M. R. Wasielewski, V. B. Prakapenka, D. V. Talapin and R. D. Schaller, Nano Lett., 2018, 18, 6948-6953.

53 O. Erdem, M. Olutas, B. Guzelturk, Y. Kelestemur and H. V. Demir, J. Phys. Chem. Lett., 2016, 7, 548-554.

54 E. V. Shornikova, D. R. Yakovlev, L. Biadala, S. A. Crooker, V. V. Belykh, M. V. Kochiev, A. Kuntzmann, M. Nasilowski, B. Dubertret and M. Bayer, Nano Lett., 2020, 20, 13701377.

55 D. Kudlacik, V. F. Sapega, D. R. Yakovlev, I. V. Kalitukha, E. V. Shornikova, A. V. Rodina, E. L. Ivchenko, G. S. Dimitriev, M. Nasilowski, B. Dubertret and M. Bayer, Nano Lett., 2020, 20, 517-525.

56 A. Esser, E. Runge, R. Zimmermann and W. Langbein, Phys. Rev. B: Condens. Matter Mater. Phys., 2000, 62, 8232.

57 M. Combescot and S.-Y. Shiau, Excitons and Cooper pairs: two composite bosons in many-body physics, Oxford University Press, 2015.

58 A. M. Jones, H. Yu, N. J. Ghimire, S. Wu, G. Aivazian, J. S. Ross, B. Zhao, J. Yan, D. G. Mandrus, D. Xiao, et al., Nat. Nanotechnol., 2013, 8, 634.
59 D. Andronikov, V. Kochereshko, A. Platonov, T. Barrick, S. Crooker and G. Karczewski, Phys. Rev. B: Condens. Matter Mater. Phys., 2005, 72, 165339.

60 P. P. Jha and P. Guyot-Sionnest, ACS Nano, 2009, 3, 1011-1015.

61 V. I. Klimov, Annu. Rev. Phys. Chem., 2007, 58, 635-673.

62 J. Feldmann, G. Peter, E. Göbel, P. Dawson, K. Moore, C. Foxon and R. Elliott, Phys. Rev. Lett., 1987, 59, 2337.

63 H. Akiyama, S. Koshiba, T. Someya, K. Wada, H. Noge, Y. Nakamura, T. Inoshita, A. Shimizu and H. Sakaki, Phys. Rev. Lett., 1994, 72, 924-927.

64 D. P. Morgan and D. F. Kelley, J. Phys. Chem. C, 2019, 123, 18665-18675.

65 C. Robert, D. Lagarde, F. Cadiz, G. Wang, B. Lassagne, T. Amand, A. Balocchi, P. Renucci, S. Tongay, B. Urbaszek, et al., Phys. Rev. B, 2016, 93, 205423.

66 S. Ayari and S. Jaziri, Phys. Status Solidi B, 2019, 256, 1800682.

67 H. H. Fang, B. Han, C. Robert, M. A. Semina, D. Lagarde, E. Courtade, T. Taniguchi, K. Watanabe, T. Amand, B. Urbaszek, M. M. Glazov and X. Marie, Phys. Rev. Lett., 2019, 123, 067401.

68 E. Vanelle, M. Paillard, X. Marie, T. Amand, P. Gilliot, D. Brinkmann, R. Lévy, J. Cibert and S. Tatarenko, Phys. Rev. B: Condens. Matter Mater. Phys., 2000, 62, 2696.

69 G. Finkelstein, V. Umansky, I. Bar-Joseph, V. Ciulin, S. Haacke, J.-D. Ganiere and B. Deveaud, Phys. Rev. B: Condens. Matter Mater. Phys., 1998, 58, 12637.

70 B. Patton, W. Langbein and U. Woggon, Phys. Rev. B: Condens. Matter Mater. Phys., 2003, 68, 125316.

71 M. Califano, A. Franceschetti and A. Zunger, Phys. Rev. B: Condens. Matter Mater. Phys., 2007, 75, 115401.

72 J.-h. Wang, G.-j. Liang and K.-f. Wu, Chin. J. Chem. Phys., 2017, 30, 649-656.

73 S. A. Cherevkov, M. V. Artemyev, A. V. Prudnikau and A. V. Baranov, Phys. Rev. B: Condens. Matter Mater. Phys, 2013, 88, 041303.

74 A. Hichri, I. Ben Amara, S. Ayari and S. Jaziri, J. Appl. Phys., 2017, 121, 235702.

75 N. S. Rytova, arXiv preprint arXiv:1806.00976, 2018.

76 A. Chernikov, T. C. Berkelbach, H. M. Hill, A. Rigosi, Y. Li, O. B. Aslan, D. R. Reichman, M. S. Hybertsen and T. F. Heinz, Phys. Rev. Lett., 2014, 113, 076802.

77 P. Cudazzo, I. V. Tokatly and A. Rubio, Phys. Rev. B: Condens. Matter Mater. Phys., 2011, 84, 085406.

78 F. García Flórez, A. Kulkarni, L. D. A. Siebbeles and H. T. C. Stoof, Phys. Rev. B, 2019, 100, 245302.

79 Y. Louyer, L. Biadala, P. Tamarat and B. Lounis, Appl. Phys. Lett., 2010, 96, 203111.

80 S. R. Stock, Microcomputed tomography: methodology and applications, CRC press, 2018.

81 A. Esser, R. Zimmermann and E. Runge, Phys. Status Solidi $B, 2001,227,317-330$.

82 G. V. Astakhov, V. P. Kochereshko, D. R. Yakovlev, W. Ossau, J. Nürnberger, W. Faschinger and G. Landwehr, Phys. Rev. B: Condens. Matter Mater. Phys., 2000, 62, 1034510352. 
83 Z. Hens and I. Moreels, J. Mater. Chem., 2012, 22, 10406.

84 P. Geiregat, R. Tomar, K. Chen, S. Singh, J. M. Hodgkiss and Z. Hens, J. Phys. Chem. Lett., 2019, 10, 3637-3644.

85 M. Combescot and J. Tribollet, Solid State Commun., 2003, 128, 273-277.

86 M. Combescot and O. Betbeder-Matibet, Phys. Rev. B: Condens. Matter Mater. Phys., 2009, 80, 205313.

87 S. Ithurria and D. V. Talapin, J. Am. Chem. Soc., 2012, 134, 18585-18590.

88 R. A. Sergeev, R. A. Suris, G. V. Astakhov, W. Ossau and D. R. Yakovlev, Eur. Phys. J. B, 2005, 47, 541-547.
89 E. Courtade, M. Semina, M. Manca, M. M. Glazov, C. Robert, F. Cadiz, G. Wang, T. Taniguchi, K. Watanabe, M. Pierre, W. Escoffier, E. L. Ivchenko, P. Renucci, X. Marie, T. Amand and B. Urbaszek, Phys. Rev. B, 2017, 96, 085302 .

90 W. Shan, J. Song, H. Luo and J. Furdyna, Phys. Rev. B: Condens. Matter Mater. Phys., 1994, 50, 8012.

91 S.-H. Wei and A. Zunger, Phys. Rev. B: Condens. Matter Mater. Phys., 1999, 60, 5404.

92 Y. Kim, M. Klein, S. Ren, Y. Chang, H. Luo, N. Samarth and J. Furdyna, Phys. Rev. B: Condens. Matter Mater. Phys., 1994, 49, 7262 . 